\section{ECONOMÍA DE LOS}

\section{CONTENIDOS ONLINE:}

\section{PERIÓDICOS ONLINE Y}

\section{EBOOKS}

\section{Julio Larrañaga Rubio}

\section{Profesor Titular}

Facultad de Ciencias de la Información. Departamento de Análisis Económico. Universidad Complutense de Madrid. Ciudad Universitaria, Madrid 28040 (España) - Email: juliolr03ehotmail.com

\section{Resumen}

El uso de las nuevas tecnologías está implicando un profundo cambio en los hábitos de consumo y acceso a los contenidos. El consumidor se está alejando de las formas tradicionales de consumo, por lo que se rompen los modelos tradicionales de distribución y producción de estos productos. En el artículo se analizan las principales pautas del mercado de contenidos digitales, profundizando la exposición en la situación de dos mercados: los periódicos y los libros.

\section{Palabras clave}

Industria de los periódicos, editor, edición digital, editorial, contenidos online, periódicos online, ebooks, distribución online

Key Words

Newspaper industry, publishers, digital edition, publishing, online contents, online newspapers, e-books, online distribution

\section{Abstract}

The use of new technologies has farreaching implications in consumer patterns and in the access to contents, whereby the consumer is taking distance from former consumption patterns and this in turn is eroding the conventional production and distribution models for these products. This paper studies the main market traits of digital contents and highlights specially the newspaper and the book market. 


\section{Introducción}

Las nuevas tecnologías están determinando un nuevo sistema de producción, distribución y consumo de los contenidos y estos nuevos sistemas suponen un nuevo modelo de negocio Se va a analizar el mercado de un producto online cuyo consumo está muy extendido en España, los periódicos online, y se compara con el mercado de los ebooks, un producto que todavía no ha consolidado su consumo en el mercado de contenidos online de nuestro país.

\section{Objetivos}

Analizar el mercado de los contenidos online y sus nuevas posibilidades de nego- cio, especialmente en el caso de los periódicos online y de los ebooks.

\section{Metodología}

Análisis comparativo de los datos estadísticos y de la información económica disponible. Se utiliza la información estadística que proporcionan: EIAA (European Interactive Advertising Association), AIMC (Asociación para la Investigación de Medios de Comunicación), FGEE (Federación de
Gremios de Editores de España, Fundación BBVA y PwC (PricewaterhouseCoopers). Así como distintos informes sobre los mercados de contenidos online y sobre la industria de los periódicos y de los libros.

\section{Situación actual del mercado $y$ previsiones de crecimiento a medio plazo a nivel mundial $y$ en España de la industria de los periódicos $y$ de la industria editorial}

En primer lugar se analizan las previsiones de crecimiento de estas industrias exponiendo las principales conclusiones que proporciona el informe "Global Entertainment and Media Outlook: 2008-2012" (GEMO) elaborado por la consultora Pri- 
cewaterhouseCoopers (PwC). Esta consultora estima que el sector de Ocio y Medios de Comunicación tendrá un crecimiento medio anual a nivel mundial del $6,6 \%$ en dicho periodo de tiempo, hasta llegar a los 2,2 billones de dólares en el 2012 (PwC, 2008b: 2). En el informe se realizan previsiones de crecimiento de 14 industrias: cinematográfica, periódicos, libros, publicidad y acceso a Internet, información empresarial, videojuegos, televisión convencional, televisión de pago, revistas, radio, publicidad exterior, deportes, parques temáticos y de atracciones y casinos. Aunque en la actualidad la mayoría de los ingresos del sector de Ocio y Medios de Comunicación corresponde a los ingresos tradicionales del sector, la distribución digital es el principal generador de los nuevos ingresos. También se especifica en este informe que el menor nivel de renta disponible de los consumidores provocado por la crisis económica no afecta de la misma forma al consumo de los distintos bienes que se asocian con la industria de entretenimiento y ocio (PwC, 2008a: 3 y 4).

Por otra parte, la mayor penetración de la banda ancha está implicando que la compañías de medios diversifiquen los canales de distribución de sus contenidos guiándose por la premisa de "anytime, anywhere" para, actuando de esta forma, poder satisfacer las necesidades de sus clientes. Pero la migración hacia los formatos digitales de los contenidos ya está afectando de forma negativa a los formatos físicos. Un claro ejemplo de ello es la disminución de ingresos del 4,1\% del mercado discográfico en el 2007, o la reducción del 0,2\% experimentada también en ese mismo año por la inversión publicitaria en prensa tradicional, o del 1,1\% en la radio (PwC, 2008a:8). La banda ancha permite nuevas posibilidades de ocio y entretenimiento que "están impactando de forma decisiva en los modelos de negocio establecidos y abriendo nuevas ventanas a nuevos modelos de ocio digital” (PwC, 2008a:3). Pero se está produciendo una fragmentación de las audiencias motivada por los cambios en los hábitos de los consumidores, que reparten su atención entre las ventanas clásicas de distribución y las nuevas. Esta fragmentación de ventanas implica que ahora más que nunca se puede decir que "Content is King" ( $\mathrm{PwC}$, 2008a:3)

Aunque en el año 2007 la distribución de contenidos digitales sólo represento el 5\% de los ingresos del sector, se estima que este tipo de formatos supondrá el 11\% de la facturación total en el año 2012 (PwC, 2008a:8).

En España, la industria de ocio y entretenimiento crecerá entre los años 2008 y 2012 a una media anual del 7,9\% (PwC, 2008b:1), por lo tanto el crecimiento previsto en España es más elevado que la media mundial, concretamente 1,3 puntos porcentuales más, y su facturación pasará de 38.799 millones de dólares en el año 2008 a 51.947 en el 2012. Los sectores que 
tendrán una mayor expansión son: la televisión por satélite, cable e IPTV con un crecimiento medio anual del 16,5\% e Internet con el 12,8\%. En cambio se estima que las industrias discográficas y cinematográficas sufrirán unas caídas medias del $4,4 \%$ y del $1,1 \%$, respectivamente. Concretamente, la edición de libros se estima que crecerá en el periodo citado a una tasa anual media del 2,1\%, y la de periódicos del 2,9\% (PwC, 2008b:2). Por lo tanto, la edición de libros crecerá en el periodo a una tasa que es el $25 \%$ de la tasa media de crecimiento del sector de ocio y medios de comunicación en España. La edición de periódicos crecerá el 37\% de la tasa media, siendo los sectores que tienen estimadas unas tasas de crecimiento más reducidas de los 15 analizados, excluidos los dos anteriormente mencionados, cuyo crecimiento se prevé negativo. A continuación, se comentan las principales características $y$ previsiones incluidas en el "Global Entertainment and Media Outlook: 2008-2012" de las industria de edición de libros y de periódicos.

En el informe de $\mathrm{PwC}$, se engloban dentro de los ingresos del mercado editorial los volúmenes de gasto de los consumidores, organismos públicos y escuelas en la compra de libros impresos, electrónicos y audio-books y se excluyen los libros profesionales. Las principales previsiones y drivers del mercado editorial a nivel mundial son:

- Se estima que el mercado crecerá a una tasa media anual del 2,8\% en el periodo
2008-2012. Por su parte, en España la tasa será menor, del 2,1\%.

- Los ebooks tendrán un elevado crecimiento, hasta llegar a suponer el 5,2\% del total de ingresos de la industria en el 2012.

- Todos los drivers del mercado editorial que se mencionan en el informe de $\mathrm{PwC}$ están relacionados con la digitalización y los libros electrónicos, lo que refleja la importancia que tendrán en un futuro próximo.

- Se afirma que los menores ingresos que obtendrá la industria por la venta de libros comerciales debido a la crisis económica se podrán compensar con unas mayores adquisiciones de sus versiones electrónicas. Además, la mayor oferta de ebooks disponibles en la actualidad, debida al lanzamiento de versiones digitales de las grandes editoriales como Penguin, HaperCollins y Rabdom House, garantizan que el lector pueda encontrar de manera muy fácil el libro electrónico que le interesa.

- No se estima que los ebooks canibalicen la venta de libros físicos, ya que la impresión por parte del lector de ellos no es eficiente, debido a los costes de papel y tinta, además de las reticencias de muchos lectores a la lectura de los textos en pantalla, por lo menos de los volúmenes de muchas páginas.

- Se potenciarán nuevos modelos de negocio en el sector, como por ejem- 
plo, la impresión bajo demanda, impulsados por un mayor volumen de libros digitalizados. Este tipo de impresión se afirma que "ya ha alterado sustancialmente las reglas de negocio establecidas en segmentos como los libros de carácter educativo" (PwC, 2008a: 28).

- La aparición en el mercado de nuevos dispositivos para la lectura de ebooks, como el Kindle de Amazon y el Reader de Sony han supuesto una auténtica revolución, ya que la nueva tecnología que utilizan permiten una lectura mucho más cómoda de los textos en las pantallas del ordenador. Por ello, las empresas del sector han visto una “oportunidad en esta tecnología y las grandes editoriales están desarrollando la oferta que pueda satisfacer la demanda de lectura en este nuevo formato" (PwC, 2008a:28)

- En relación a los audio books, se especifica la estrategia seguida por los editores de ofrecer la posibilidad de descargarlos sin Gestión de Derechos Digitales (DRM).

Por otra parte, el mercado español de la edición de periódicos se estima que crecerá en el periodo 2008-2012 a una media anual del 2,9\%, es decir una tasa superior a la media mundial, 2,2\%. En el mercado de edición de periódicos $\mathrm{PwC}$ incluye los ingresos por compra de ejemplares de los consumidores tanto en los puntos de venta como por suscripción, así como los ingresos obtenidos por la publicidad en cual- quiera de los soportes. Las principales estimaciones y drivers de este mercado a nivel mundial son:

- Se estima que los ingresos por circulación crecerán a nivel mundial durante el periodo a una media anual del 0,9\%. Además se prevé que habrá una disminución en la circulación de los periódicos de pago en Canadá, Estados Unidos y Europa, mientras se producirá un aumento de los volúmenes de circulación en Asia y Latinoamérica.

- El ingreso más importante de la industria seguirá siendo la publicidad impresa, con un crecimiento anual medio del $1,8 \%$. Aunque la situación económica implicará en los primero años analizados por $\mathrm{PwC}$ un menor volumen de inversión publicitaria en los periódicos impresos, al final del periodo se producirá una cierta recuperación de estos ingresos. Además, la migración de los lectores de las ediciones en papel a las digitales "impulsará el aumento de la publicidad online a la vez que recortará la circulación de ejemplares" (PwC, 2008a:26). Por lo tanto, se producirá una canibalización de las versiones impresas de los periódicos por las online.

- Los ingresos de los periódicos digitales se estima que crecerán a una media anual del 19,3\%, llegando a superar en el año 2012 el 10\% del total de ingresos del mercado de los periódicos. 


\section{Cambios en la estructura de los mercados de contenidos provocados por el uso de las Nuevas Tecnologías}

El uso masivo de Internet está implicando grandes cambios tanto en las empresas de contenidos, como en las formas de consumo de dichos contenidos por los consumidores. Estas alteraciones están provocando una "economía interconectada, basada en la información, que ha dado lugar a una nueva relación entre el cliente, los productos o servicios y el canal de distribución" (Valor y Hess, 2001:32). En la distribución de los contenidos físicos el proveedor de dichos contenidos está situado en el extremo opuesto del consumidor, siendo los intermediarios el canal de distribución que le permiten acceder al cliente, pero en el caso de la distribución online este canal tiene la posibilidad de capturar un porcentaje muy elevado del valor percibido por el cliente, e inclusive en algunos casos "es posible que dicho valor no llegue a manos del proveedor de contenidos, mientras que en otros, es solamente la asociación exclusiva de proveedores de contenido y canal la que permite al canal sobrevivir" (Valor y Hess, 2001:32). Dentro del término valor percibido por el cliente, estos autores engloban tanto el pago directo que realiza el consumidor -incluyendo dentro de este pago el importe de la factura por conexión a la Red- como el efecto de la influencia de la publicidad que recibe el consumidor en el caso de que se suministre con el contenido online.

Cassiman y Sieber afirman que la creación de valor se produce cuando "la predisposición a pagar por un determinado producto o servicio es superior al coste de proveer dicho producto o servicio" (Cassiman y Sieber, 2001:15), es decir, cuando la disponibilidad a pagar de los consumidores es superior al coste de los recursos utilizados para la producción de dicho bien o servicio. En Internet se presenta una situación de elevada competencia entre los portales, proveedores de acceso a la Red y empresas de contenidos online por intentar conseguir un mayor porcentaje del valor percibido por el consumidor. Además "la rentabilidad de las empresas que compiten de manera aislada está muy en entredicho, excepto en los casos de dominio del sistema en presencia de externalidades de red (Microsoft) o de monopolio histórico (operadores tradicionales de telefonía)" (Valor y Hess, 2001:41). Por ello, las distintas empresas se integran verticalmente para conseguir tener garantizados el suministro de los servicios, o el acceso a canales que las pueden discriminar. Por ejemplo, las compañías de contenidos movidas por el 
objetivo de asegurarse el acceso a sus clientes potenciales, intentan conseguir acuerdos con un horizonte temporal de largo plazo con la empresa propietaria de los bucles locales de teléfonos en los mercados en los que dicho bucle pertenece a un monopolio.

Por lo tanto, las empresas se integran verticalmente para optimizar sus resultados. Esta integración se produce entre las empresas de contenidos, los portales, los proveedores de acceso a la Red y los proveedores de contenidos. Un ejemplo de este proceso de integración con el objetivo de que la nueva empresa creada esté presente en todas las etapas de los que se denomina por parte de Valor y Hess como "cadena de valor online", es el caso de American Online y Time Warner. Se puede resumir su cadena de valor online (que son las etapas de creación de valor de una empresa) de la siguiente forma: la nueva empresa que integra a ambas proporciona "contenido a través de empresas de publicaciones... filmación... entretenimiento... música y video interactivo. Estos contenidos se agregan a través de su portal...Llegan al consumidor mediante su propio proveedor de acceso a Internet... así como sus propias redes y sistemas de cable" (Cassiman y Sieber, 2001:15).

Es decir, las TIC están provocando un profundo cambio en las estructuras de los mercados y "está alterando de forma significativa el modelo de funcionamiento de algunas empresas y sectores, no sólo redu- ciendo de forma significativa el tiempo y coste de procesamiento de todo tipo de información sino modificando el modo en que empresas y mercados gestionan el flujo de bienes y servicios a través de sus cadenas de valor (López y Arroyo, 2006:361). El principal efecto que provocan las nuevas tecnologías en las empresas se debe a las externalidades de red, que también se denominan efectos de red o economías de escala desde el lado de la demanda “y de hecho es tal su importancia en los mercados electrónicos que algunos autores... afirman que la esencia de la economía digital se encuentra precisamente en una extraordinaria presencia de dichas externalidades" (López y Arroyo, 2006:21). Una externalidad de red se produce cuando "el valor de un producto o servicio para un usuario dependa no sólo del producto en sí mismo, sino del número de usuarios que utilizan dicho producto o servicio (Fuentelsaz, Maicas y Polo, 2002:10).

Los términos de externalidades y efectos de red, son conceptos distintos, aunque muchas veces se utilizan como sinónimos. López y Arroyo señalan que para que exista una externalidad se "debe producir un fallo en el mercado de modo que los participantes en el mismo no sean capaces de internalizar el beneficio generado por un nuevo actor" (López y Arroyo, 2006:22). Por ese motivo indican que si hay externalidades "el valor para un nuevo usuario es menor que el valor social que este aportaría, se alcanzará un tamaño de la red inferior al 
tamaño óptimo" (López y Arroyo, 2006:22). Pero si se redefinen los derechos de propiedad, y nos movemos con redes esponsorizadas, entonces "no hay obstáculos para que el propietarios de la misma internalice estos efectos, de modo que existirían efectos de red, pero no externalidades" (López y Arroyo, 2006:22).

Shapiro y Varian afirman que "hay una diferencia fundamental entre la nueva y la antigua economía: la vieja industria estaba impulsada por las economías de escala; la nueva economía de la información está impulsada por la economía de las redes" (Shapiro y Varian, 2000:165). Es por esto que la economía industrial estaba poblada de oligopolios y en cambio la economía de la información está poblada de monopolios temporales, por lo tanto, la economía de las redes, reduce el grado de competencia de los mercados.

Internet afecta en un grado muy elevado la estructura de los mercados de bienes y servicios, sus efectos se pueden resumir en los siguientes puntos (Porter, 2001:5):

- Los costes más bajos generan una mayor eficiencia.

- La reducción de los costes de búsqueda de los consumidores afecta a su disponibilidad de pago.

- Surgen nuevas oportunidades en la empresa para la diferenciación de sus productos.

- Se producen cambios en las políticas de precios.
- Se reducen las barreras de entrada a la industria.

Es decir, la Red afecta a la empresa tanto desde el punto de vista de la demanda como de los costes, provocando una transformación de los mercados, especialmente en los sectores en los que el "tratamiento de la información es clave: los contenidos (sector de entretenimiento), la comunicación de la información (sector de las telecomunicaciones), y sus infraestructuras (sectores de hardware, software y electrónica)" (Cassiman y Sieber, 2001:13), todos estos sectores están inmersos en un "proceso de convergencia sectorial en el cual han surgido nuevos sectores con dinámicas competitivas diferentes" (Cassiman y Sieber, 2001:13).

Inicialmente se podría pensar que Internet al facilitar la transparencia de los mercados implicaría unos precios más bajos de los productos, ya que las empresas que venden sus productos al precio más bajo son indexadas en los motores de búsqueda del menor precio. Por lo tanto, habría un proceso de competencia entre las distintas empresas que las llevaría a igualar sus precios con sus costes de oportunidad. Según Cassiman y Sieber, el nivel de transparencia de los mercados existente en la Red ha llevado a la hipótesis de que la competencia entre empresas tendría lugar en "forma de competencia de Bertrand, en la que las empresas compiten meramente en precios, por lo que la situación de equilibrio prevé precios más bajos con una dispersión me- 
nor de precios y, por tanto, con una baja rentabilidad sectorial" (Cassiman y Sieber, 2001:21). En cambio, la evidencia empírica muestra que aunque existen precios más bajos en Internet, todavía sigue existiendo un cierto grado de dispersión de precios motivada por "la existencia de información asimétrica, el hecho de que en realidad los costes de búsqueda, aunque se hayan reducido, siguen existiendo, y la heterogeneidad de los productos" (Cassiman y Sieber, 2001:22).

Por otro lado, Internet permite la implantación de nuevos mecanismos en la fijación de precios, como por ejemplo las subastas, y también facilita la personalización de los productos. Estos dos efectos incrementan la disponibilidad a pagar de los consumidores y además actúan como una barrera de entrada endógena, dificultando la competencia de nuevas empresas (Cassiman y Sieber, 2001:22 y 23). Un hecho que fomenta la disparidad de precios en Internet, es la política de precios seguida por las tiendas que también tienen una presencia física, la evidencia empírica demuestra que estas empresas venden sus productos más caros en la Red que aquellas tiendas que sólo tienen una existencia online. Las primeras fijan unos precios superiores con el fin de evitar que las ventas online canibalicen el negocio de su tiendas tradicionales (Friberg, Ganslandt y Sandström, 2001:16).

\section{El consumo online de los bienes culturales $y$ de entretenimiento}

El progresivo grado de implantación de Internet en la sociedad está provocando un profundo cambio en las pautas de consumo de los productos culturales y en las distintas actividades de ocio del usuario de la Red, no sólo porque al tener que repartir los consumidores su tiempo de ocio entre las diversas alternativas el mayor uso de Internet implique un menor tiempo disponible para el consumo de otros productos, lo que provoca un claro efecto sustitución, sino porque también se está produciendo en el consumidor potencial un cambio del consumo de los productos offline por sus equivalentes online (Larrañaga, 2009:13). Se estima que el consumo de los bienes culturales y de entretenimiento es el más afectado por el desarrollo de la Sociedad de la Información (Pablo, Mañas y Cuadrado, 2006:62). Desde el punto de vista de los consumidores, las TIC producen dos efectos claros en el consumo de cualquier tipo de bien., en primer lugar facilitan una "gestión más eficiente de sus recursos al liberar tiempo y dinero para otros usos" (Pablo, Mañas y Cuadrado, 2006:61). Esto es debido a que las nuevas tecnologías reducen la asimetría de información que 
existe entre los consumidores y los productores, permitiendo que los primeros obtengan de forma fácil información relacionada con los distintos precios y calidades de los productos disponibles, aunque este efecto es muy difícil valorarlo económicamente. Los mayores niveles de información disponible también afectan a los productores potenciales, y por ello se produce de una forma más fácil la entrada de nuevas empresas que quieren satisfacer las necesidades de los nichos de mercado existentes. Esto a su vez provoca una mejora en la eficiencia por los mayores niveles de competencia en los mercados, y lógicamente, también se consigue una mayor eficiencia por el mayor conocimiento que tienen los consumidores sobre las distintas características de los distintos bienes y servicios existentes en el mercado.

El incremento del número de empresas que existen en el mercado aumenta la oferta y los consumidores pueden elegir entre un mayor número de bienes y servicios. Este hecho se produce siempre que no existan los costes denominados como "lock-in", los costes de cambio de proveedor que aparecen cuando el usuario queda vinculado a una determinada tecnología debido a que los costes de cambio son muy elevados. El lock-in de los clientes es la norma en la economía de la información "debido a que la información se almacena, se manipula y se comunica usando un sistema que consta de múltiples piezas de hardware y software y debido a que se requiere un adiestramien- to especializado para usar estos sistemas concretos" (Shapiro y Varian, 2000:111). Por lo tanto, el lock-in aleja al mercado de las condiciones de competencia perfecta, ya que "refuerza la posición de una tecnología ya instalada como consecuencia de los costes, tanto individuales como sociales, que implica la adopción de un nuevo estándar tecnológico" (López y Arroyo, 2006:23). Además, la empresa poseedora de una determinada tecnología suele ampliar su posición privilegiada potenciando el mercado de productos complementarios, con ello consigue fortalecer su tecnología y logra que aumente su grado de expansión, lo que facilita que se fortalezca el efecto lock-in ya que al existir una mayor cantidad de productos complementarios "se expande la base de clientes de la tecnología lo que a su vez genera una mayor cantidad de complementarios" (López y Arroyo, 2006:28). Por otra parte, la implantación de las TIC en los hogares está provocando un hecho muy relevante en los mercados de los bienes culturales y de entretenimiento ya que ni la inmediatez de la información, la mejora de la calidad de las comunicaciones o los cambios en las forma de entretenimientos que disfrutan los consumidores "se están traduciendo en un aumento paralelo en el gasto que realizan en estas partidas, a pesar de que la importancia que tienen en sus vidas es claramente creciente" (Pablo, Mañas y Cuadrado 2006:61).

Con anterioridad a la incorporación de forma masiva de las TIC en los hogares, los 
consumidores percibían los servicios de comunicación, información y entretenimientos, como servicios distintos, suministrados además por diversas compañías. En la actualidad gracias a la convergencia tecnológica estos servicios son ofrecidos a los consumidores por varias empresas, no sólo por las que antes los suministraban de forma habitual, sino que también han aparecido nuevas empresas competidoras, lo que ha implicado que "la posición de los consumidores haya mejorado tanto en términos de variedad de la oferta como en capacidad de negociación” (Pablo, Mañas y Cuadrado, 2006:62), se considera que esté hecho es el segundo gran efecto de las TIC en el consumo de las familias.

En relación a los bienes y servicios culturales ligados a la Sociedad de la Información se están produciendo cambios cada vez más profundos tanto en relación a las cantidades consumidas de los mismos, como en la forma de utilización de dichos productos. Estos bienes y servicios estás experimentando una "continuada reducción de sus coste medio efectivo para el consumidor lo que está produciendo una rápida expansión en su consumo medido en unidades físicas" (Pablo, Mañas y Cuadrado, 2006:67), esta reducción del coste medio para el consumidor está motivada tanto por una disminución del precio de estos productos en términos reales, como por la posibilidad que tienen las familias actualmente de un consumo paralelo de dichos productos a un coste nulo o prácticamente nulo. Por esta razón "no se observan cambios excesivamente importantes en el gasto realizado por las familias en los bienes y servicios culturales ligados a la Sociedad de la Información” (Pablo, Mañas y Cuadrado, 2006:67).

Por lo tanto, el mayor uso de las TIC en el hogar ha permitido a las familias "un mayor acercamiento... al consumo gratuito de bienes culturales y de entretenimiento" (Pablo, Mañas y Cuadrado, 2006:67), ya sea por medio del uso por parte de los consumidores de los distintos servicios que se proporcionan gratis a través de Internet, como por los sistemas de intercambio de ficheros.

Las empresas productoras de bienes de información online suministran en un primer momento estos bienes de forma gratuita para que el consumidor se habitúe a su consumo. Sin embargo, ya que estas empresas tienen una estructura de coste muy peculiar, este suministro gratuito de sus productos no eleva sus costes marginales. Una empresa cuando produce bienes de información incurre en un coste elevado al producir la primera unidad del bien, lo que se denomina como "el coste de la primera copia”. Pero los costes de aumentar la producción son muy reducidos. Por lo tanto, incurre en unos elevados costes fijos al producir el primer ejemplar del bien, pero debido a que los costes variables son muy reducidos, cuando mayor sea su volumen de producción, menor es el coste 
medio, es decir hay unas elevadas economías de escala (Shapiro y Varian, 2000:21).

Una característica de los bienes de información es que su producción implica unos costes fijos muy altos, pero también tiene unos costes marginales muy bajos, "la información es cara de producir pero barata de reproducir... El coste de producir el primer ejemplar de un bien de información puede ser considerable, pero el coste de producir (o reproducir) copias adicionales es insignificante (Shapiro y Varian, 2000:3). Estos autores acotan el término de bien de información de una forma muy amplia, "entenderemos por información cualquier cosa que pueda ser digitalizada codificada como un conjunto de bits-" (Shapiro y Varian, 2000:2). Es decir, se engloban dentro del término tanto los periódicos, como los libros, la música, las películas o las bases de datos. Y afirman que el aspecto más relevante de los bienes de información suministrados online es que su "coste de producción esta determinado por el coste de la primera copia...La información que llega por una red en forma digital presenta el problema de la primera copia de forma exacerbada: una vez que se ha producido la primera copia de la información, las copias adicionales no cuestan prácticamente nada" (Shapiro y Varian, 2000:20).

La estructura de los costes fijos y variables de la producción de bienes de información es muy particular. En primer lugar, un porcentaje muy elevado de los costes fijos empleados en la producción de estos bienes son "costes enterrados, es decir costes no son recuperables por mucho que se detenga la producción...Además de los costes enterrados de la primera copia, los costes de comercialización y promoción son muy importantes" (Shapiro y Varian, 2000:21), como la atención del consumidor es un bien escaso las empresas vendedoras de contenido tienen que invertir en la comercialización de sus nuevos productos para poder conseguir atraer la atención de sus clientes potenciales. Por otra parte, también los costes variables tienen una estructura peculiar ya que "el coste de producir copias adicionales generalmente no aumenta, incluso en el caso de que se hagan muchísimas copias" (Shapiro y Varian, 2000:21). Esto se debe a que son empresas que no se enfrentan con restricciones de capacidad importantes y duraderas. En la producción de bienes de información se pueden "producir multitud de copias a costes unitarios prácticamente constantes. No hay límites de capacidad naturales para realizar copias adicionales" (Shapiro y Varian, 2000:22).

Esta estructura de costes determina que en el mercado de los bienes de información sólo hay dos estructuras de mercado sostenibles:

- "El modelo de empresa dominante, pueda producir o no el mejor producto, pero gracias a su tamaño y a sus economías de escala, disfruta de costes menores que sus rivales más pequeños” 
(Shapiro y Varian, 2000:24). Un ejemplo muy claro de ello sería Microsoft.

- "En un mercado de productos diferenciados tenemos una serie de empresas que producen el mismo tipo de información, pero con diferentes variedades. Esta es la estructura de mercado más típica de los bienes de información” (Shapiro y Varian, 2000:24), y está es la estructura del sector editorial, de la televisión, del sector cinematográfico, de la industria de los periódicos y el de algunas empresas de software.

La estrategia que debe seguir la empresa depende del sector en el que esté ubicada, si se encuentra dentro de un sector con productos diferenciados, debe diferenciar su producto, y para ello debe darle valor a la información bruta, a fin de diferenciarse de la competencia. Si está en un sector en el que existe una empresa dominante, la empresa debe centrarse en conseguir ser líder en costes, aprovechando de forma óptima las economías de escala y de alcance.

En la producción de bienes de información la clave para reducir los costes medios es aumentar el volumen de ventas, señalan Shapiro y Varian, y ponen como ejemplo de ello a la empresa Reuters que vende las mismas "parcelas de información una y otra vez. La compañía evita la trampa de que su producto estelar se convierta en mercancía organizándola de manera que sea útil para sus clientes, con lo que diferencia su producto del de la competencia" (Shapiro y
Varian, 2000:28). La empresa consigue el liderazgo en costes añadiendo valor a la información bruta. Además, como Internet facilita la personalización tanto de los productos como de los precios, permite que la empresa ofrezca distintos productos en función de las distintas necesidades de sus consumidores, con ello la empresa "crea un producto que sea único para así poder basar el precio en el valor que se ofrece al consumidor en lugar de basarlo en los precios que fija la competencia" (Shapiro y Varian, 2000:49).

Por otra parte, dado que la información es un bien de experiencia, es decir que para saber como es, hay que pasar previamente por la experiencia de probarlo, las empresas productoras de bienes de información suelen distribuir muestras gratuitas a través de Internet para acostumbrar al consumidor a su consumo. La estructura de coste de estos bienes determina que a la empresa no le cueste prácticamente nada distribuir estos productos "en los bienes de información, las copias resultan ser gratis tanto para el productor como para el consumidor" (Shapiro y Varian, 2000:21)

Pero el consumo de productos culturales y de entretenimiento a coste cero en las familias españolas "no se debe exclusivamente a la adquisición de productos y servicios gratuitos. El acceso a canales clandestinos y la utilización de medios de reproducción y distribución de difícil regulación supone también una forma importante de consumo" (Pablo, Mañas y Cuadrado, 
2006:68). De forma tradicional las barreras tecnológicas han ejercido un papel tan importante en la defensa de los derechos de propiedad intelectual como las regulaciones legales. Pero en la actualidad, tanto Internet como los métodos digitales de almacenamiento han implicando un cambio en la forma en que estas barreras han actuado tradicionalmente $\mathrm{y}$ han provocado unas profundas alteraciones en los mercados especialmente en el caso de los productos de entretenimiento e información que se distribuyen online, afectando a la remuneración de las empresas productoras de dichos contenidos y a todos los aspectos económicos de la producción y distribución por medio de Internet (Fowler, 2001:33).

Las nuevas tecnologías digitales producen un cambio sustancial en los costes de las empresa editora de contenidos, afectando a sus costes de reproducción y de distribución, en "los costes de reproducción: la tecnología reduce drásticamente los costes de hacer reproducciones perfectas. Costes de distribución: la tecnología digital permite que estas reproducciones sean distribuidas de una manera rápida, fácil y barata” (Shapiro y Varian, 2000:80). La reducción de estos costes afecta al control de los derechos de propiedad, además las leyes de propiedad intelectual tienen bastantes dificultades a la hora de enfrentarse a los problemas que surgen con los contenidos digitales. En opinión de algunos autores "muchos de sus principios son válidos todavía. Lo que ha cambiado es que Internet, y la tecnología de la información en general, ofrecen nuevas oportunidades y desafíos a la hora de aplicar estos principios" (Shapiro y Varian, 2000:79), y estos autores especifican que la reducción de los costes de copia y distribución permite nuevas oportunidades de negocio a los propietarios de contenidos. Otros autores también afirman que Internet ha producido un cambio drástico del modelo de negocio de dichas empresas (Fowler, 2001:47).

Las empresas deben optimizar las posibilidades que les permiten las nuevas tecnologías, por ejemplo, los "menores costes de distribución pueden ayudar a promocionar el producto, ya que resulta barato regalar muestras. Esto es tanto más útil cuanto mayor sea la demanda por ver o usar el producto repetidas veces o por contenidos muy similares" (Shapiro y Varian, 2000:97). Por lo tanto, regalar muestras de sus productos ayuda a las empresas de contenidos online a vender más contenidos.

Las muestras de productos de información son eficaces para incrementar el mercado ya que al ser estos productos lo que se denomina como bienes de experiencia, la muestra permite que el consumidor pueda conocerlos, además debido al efecto del coste marginal cero, a la empresa le cuesta muy poco proporcionar estas muestras. La empresa productora de contenidos actúa proporcionando una versión gratuita de su producto, pero esta versión es de gama baja y "está limitada en cuanto al alcance, con- 
veniencia, calidad y/o cantidad" (Shapiro y Varian, 2000:86). Otra estrategia que puede seguir la empresa es vender productos complementarios, por ejemplo, "regalar un índice o tabla de contenidos y vender el acceso al material principal... A veces la idea es inversa: el contenido es gratuito y la organización de éste es la que cuesta dinero" (Shapiro y Varian, 2000:77).

La empresa de contenidos también debe de optimizar la posibilidad que le brinda la tecnología digital de realizar copias perfectas del original y a un coste muy reducido. Debe aprovechar este reducido coste para aumentar la cantidad de productos que distribuye y poder conseguir de esta forma una masa crítica de consumidores que le permita poder explotar las economías de escala. Shapiro y Varian ponen el ejemplo de que es más rentable tener mil consumidores que pagan un dólar por un producto cuyo costes de producción y distribución es de unos pocos centavos, que tener 100 consumidores que pagan 10 dólares por un producto cuya producción y distribución cuesta 5 dólares. Y especifican que en la actualidad los productores de contenido digital están en la misma posición en la que se encontraba la industria cinematográfica en el año 1975 con los aparatos de vídeo, inicialmente se los consideró como un producto sustitutivo, cuando en realidad son complementarios. Ambos autores afirman también que "la tendencia lógica es que los productores se preocupen demasiado por proteger su propiedad intelectual.
Lo importante es maximizar el valor de nuestra propiedad intelectual, no protegerla por protegerla" (Shapiro y Varian, 2000:92). Además, señalan que si se pierde parte de la propiedad intelectual cuando se alquila o se vende un producto, esto es solo uno de los costes de hacer negocios, y se trata de un coste de la misma forma que lo son la depreciación, la obsolescencia o las pérdidas de inventario.

Dichos autores establecen que el desafío de gestionar la propiedad intelectual consiste en maximizar el valor de nuestra propiedad, pero se presenta una disyuntiva entre mantener el control y aumentar el valor que obtiene el consumidor, “cuanto más generosos sean los términos bajo los cuales los consumidores tengan acceso al producto, más valioso les será este" (Shapiro y Varian, 2000:93). Por lo tanto, estarán dispuesto a pagar un precio mayor por él y además también querrán comprar un mayor número de consumidores nuestro producto. Pero cuando se incrementa el valor del producto se producirá un segundo efecto, que se crearán "competidores al producto en los mercados de alquiler y reventa disminuyendo las ventas de los originales, con lo que se reducen los ingresos. Y los consumidores están dispuestos a pagar menos por nuestro producto si hay disponibles productos muy parecidos, tales como copias de segunda mano" (Shapiro y Varian, 2000:93).

A través de la curva de demanda se puede reflejar esta disyuntiva a la que se enfrenta 
la empresa productora de contenidos digitales. Según Shapiro y Varian puesto que los costes de producción son insignificantes se ignoran en el análisis, siendo el objetivo de la empresa maximizar sus ingresos. Si la empresa ofrece el producto en términos más generosos, aumenta el valor que el producto tiene para el consumidor, y la curva de demanda se desplaza hacia arriba, pero obviamente en este caso se harán más copias del producto, por lo que se venderá una cantidad menor. Por lo tanto, únicamente se produce un incremento de los beneficios cuando se ofrecer el producto en unos términos más generosos "si el aumento de valor que esto significa para los consumidores del que nos podemos apropiar mediante unos precios más elevados, es mayor que la reducción de las ventas" (Shapiro y Varian, 2000:94).

Shapiro y Varian señalan que "los mecanismos para protegerse de las copias imponen unos costes a los usuarios y son muy vulnerables a las fuerzas de la competencia” (Shapiro y Varian, 2000:96). Y afirman que los distintos sistemas existentes para la protección contra las copias ilegales tienen su lugar en el caso de los productos de información, pero "es muy improbable que desempeñen un papel importante en el mercado de masas de los bienes de información debido a la necesidad de estandarizar el producto y las presiones de la competencia” (Shapiro y Varian, 2000:97). Sin embargo la realidad actual es que en las redes $\mathrm{P} 2 \mathrm{P}$ se comparten decenas de millo- nes de archivos digitales de todo tipo, inclusive "la amplia difusión de este tipo de redes se está convirtiendo en uno de los motores del desarrollo actual de la banda ancha en Internet, como pone de manifiesto el amplio porcentaje que suponen en el total de datos transferidos por los usuarios residenciales de ADSL y cable" (Pablo, Mañas y Cuadrado, 2006:69)

Por otra parte, una intensificación de la lucha contra la piratería produce distintos efectos en función de sobre que tipos de consumidores se intensifiquen las medidas de protección de la propiedad intelectual. En líneas generales se puede afirmar que un aumento de los esfuerzos en defensa de la propiedad intelectual reduce los niveles de piratería, eleva el precio de los productos y reduce el excedente del consumidor. (Harbaugh y Khemka, 2001:1). Dependiendo sobre que colectivos se intensifiquen las medidas de protección de la propiedad intelectual pueden variar los resultados de estas medidas, si estas medidas se dirigen fundamentalmente sobre los usuarios para los que el producto tienen un mayor valor, y que por lo tanto, tienen una demanda del mismo muy rígida, se producirá una elevación de los precios y un aumento de los volúmenes de piratería del producto por parte de los restantes consumidores (Harbaugh y Khemka, 2001:13). Si por el contrario, las medidas de protección se dirigen a todos los consumidores, se produce un aumento de la demanda y una reducción del precio del producto. Es decir que au- 
mentan tanto los beneficios de los propietarios de la propiedad intelectual, como el excedente del consumidor, siendo claramente muchos más positivos los efectos de lucha contra la piratería en este segundo supuesto. (Harbaugh y Khemka, 2001:19).

Desde el punto de vista de la teoría económica es necesario tener en cuenta como indica Varian que "no conviene olvidar que el copyright es el second-best solution para la provisión de la propiedad intelectual” (Varian, 2006:27). Precisa este autor que "los mismos avances tecnológicos que hacen que el contenido digital resulte barato también está ayudando a reducir el coste fijo de la creación de contenidos. Centenares de miles de personas estás suministrando gratuitamente contenido digital, desde blogs o vídeos domésticos” (Varian, 2006:27). Afirma también que la mayor disponibilidad de contenido existente servirá para aumentar la competencia y que disminuya el precio que paga el consumidor por el acceso a los contenidos. También considera que esta tendencia de reducción del precio "podría servir para contrarrestar alguna de las fuerzas que han llevado a la necesidad de mayor protección de copyright" (Varian, 2006:27).

\section{Periódicos online y ebooks: reacciones de la industria y de los consumidores}

En primer lugar se expondrán las opiniones de la industria editorial española sobre los ebooks y para ello se utilizará la información suministrada por el informe "Digitalización del libro en España” realizado por el Observatorio sobre el uso de las nuevas tecnologías en el sector cultural: Dosdoce.com y la red social Ediciona. En este informe se especifica que "todas aquellas empresas que tengan como base la gestión de contenidos y su comercialización a través de intermediarios sufrirán una transformación de su modelo de negocio" (Dosdoce.com y Ediciona, 2008:9). También se detalla una serie de sectores en los que Internet ha implicado una profunda trans- formación de su modelo de negocio: medios de comunicación, música, cine, intermediarios financieros. Y se indica que "las entidades del sector del libro (editoriales, librerías y bibliotecas, etc) no van a ser una excepción a este proceso de transformación estructural" (Dosdoce.com y Ediciona, 2008:9). Las principales conclusiones del informe son:

- La mayoría de los encuestados, el 57\%, consideran que en un futuro próximo convivirán ambos soportes de lectura. Únicamente el 15\% piensa que los ebooks se impondrán sobre los libros en papel. 
- La introducción en el mercado de dispositivos que permite una lectura fácil y cómoda de los libros electrónicos, impulsará según el $80 \%$ de los encuestados, la digitalización de los libros en nuestro país.

- Para el 69\% de los encuestados la principal decisión que tienen que tomar las editoriales ante el reto de la digitalización de sus fondos es definir el modelo de negocio de la editorial en la Red. Se indica en el informe que no solo es necesario seleccionar un proveedor que digitalice todo el fondo de la editorial a un precio competitivo sino que "estamos hablando principalmente de una transformación general de la editorial, de su estrategia de producción y de distribución, de sus fuerzas políticas de marketing y comercialización de sus libros y hasta del propio mercado" (Dosdoce.com y Ediciona, 2008:9).

- Hay una profunda divergencia entre las opiniones de los empresarios del sector europeos y los españoles en relación a cual será su principal fuente de ingresos dentro de 10 años. Según los resultados de una encuesta realizada por la organización de la edición de la Feria de Frankfurt del año 2008 a 1.000 profesionales del sector, el $40 \%$ afirman que la cifra de negocio de los ebooks superará a la de los libros en papel dentro de 10 años. Por el contrario, para el 48\% de los profesionales españoles encuestados en el LIBER 2008, los libros en papel continuarán siendo la principal fuente en el 2020, y sólo el 16\% opinan que los libros electrónicos serán la primera fuente de ingresos.

También se produce una gran diferencia entre los empresarios de ambos ámbitos geográficos en el tema de los posibles acuerdos con otras industrias para generar nuevos ingresos. Esta posibilidad no es relevante para la gran mayoría de los profesionales españoles, según la encuesta realizada en el LIBER, por lo tanto, los empresarios españoles no tienen en cuenta el potencial de ingresos que puede suponer tanto la venta de sus contenidos online en diferentes soportes, como los posibles ingresos publicitarios de sus webs. Por el contrario, el $22 \%$ de los encuestados en la Feria de Frankfurt considera como muy importante la realización de acuerdos con la industria del cine, el 18\% con la industria discográfica y el 13\% con la industria del videojuego. Claramente los profesionales españoles no optimizan las posibilidades de negocio que se presentan con las nuevas tecnologías.

- Otro claro ejemplo del alejamiento del sector español de las nuevas tecnologías es que sólo el 21\% de los encuestados por Dosdoce.com considera que el diseño de sus sitios web es una prioridad importante o muy importante, por lo que no aprovechan de forma adecuada todas las posibilidades que les permite la Web 2.0 para aumentar sus ingresos. 
Las TIC, han obligado a reconfigurar el sistema editorial, sobre todo en dos aspectos fundamentales, la edición online, un buen ejemplo sería la editorial Lulú que utiliza exclusivamente este sistema y la edición bajo demanda que permite la disponibilidad en librerías en soporte papel y encuadernadas de obras que están agotadas o fuera de mercado a petición del lector interesado. Además, la publicidad por medio de catálogos online en los que se realiza una descripción pormenorizada del producto, convierte al editor en un "infomediador" con el consumidor que cambia los hábitos de consulta tradicional y el producto (López Suárez, 2008:254)

Por otra parte, no hay una encuesta disponible referida al mercado español con una muestra los suficientemente amplia, sobre las principales ventajas que lectores de ebooks asignan a los libros electrónicos, por ello como un primer acercamiento a las actitudes del consumidor ante los libros electrónicos se expondrán las principales conclusiones del informe "2008 Global Student E-book Survey” realizada por el Ebrary, que es una plataforma de edición y distribución de ebooks. En este informe se analiza el grado de uso de los libros electrónicos y de los impresos, así como las preferencias y aptitudes hacia ellos que tienen los estudiantes de diferentes países. Este informe se basa en una encuesta que contestaron 6.492 estudiantes universitarios, de 400 instituciones y de 75 países. La mayoría de los estudiantes que respondie- ron proceden de Italia, Estados Unidos, Canadá y Hong Kong y sólo la contestaron cuatro de España. Además, la mayor parte de ellos son de licenciaturas de ciencias: ingeniería, arquitectura, empresariales, informática, educación y medicina. El 57\% de los estudiantes han respondido que disponen de ebooks en sus bibliotecas, los siguientes resultados se basan en las respuestas de estos estudiantes:

- Es un poco mayor el número de estudiantes que utiliza ebooks para los trabajos de clase o investigaciones, 2.517, que los que emplean libros impresos, 2.478. El grado de utilización de Google, u otros buscadores es algo superior, 2.517. Por su parte, diccionarios y enciclopedias online son usados por 2.206 .

Además, es muy parecido el número de estudiantes que consideran fiables a los libros impresos 2.845, que el total de ellos que califican como fiables a los ebooks, 2.796, ambos son los recursos considerados como más fiables. A continuación, se encuentran los libros de texto impresos, los diccionarios y enciclopedias impresos, los periódicos físicos, y posteriormente los periódicos online, los libros de texto online, y los diccionarios y enciclopedias online

- Cuando los estudiantes tienen la oportunidad de elegir entre las versiones física y online de un libro determinado, el 22,76\% eligen muy frecuentemente la versión online, el 28,30\% frecuen- 
temente, y algunas veces el 31,76\%. Y sólo el 2,73\% elige siempre la versión impresa.

- Las características que consideran más importantes de los libros electrónicos son (el total de respuestas son 3.039): las búsquedas (2.647), la posibilidad de acceso en todo tiempo (2.626), acceso desde fuera del campus (2.481), la posibilidad de que varios estudiantes hagan uso de un libro al mismo tiempo (2.462), el que se pueda descargar al portátil (2.438), que se pueda copiar (2.292) e imprimir (2.270). Por el contrario, las posibilidades multimedia están menos valoradas (1.353).

- Los encuestados señalan que para que se utilizasen en un mayor grado los ebooks entre los estudiantes de su carrera seria necesario que hubiese mas títulos (2.465) y un menor grado de restricciones legales para copiarlos e imprimirlos (2.073).

Otra forma de exponer las principales ventajas que tienen los ebooks para sus lectores es analizar las principales conclusiones de la "Encuesta a usuarios de la Biblioteca Virtual Miguel de Cervantes sobre hábitos de lectura" realizada por su Fundación. Esta encuesta se realizó del 15 al 30 de abril de 2008, con el objetivo de una recogida de información sobre los hábitos de uso de los usuarios de la Biblioteca Virtual para potenciar su papel y detectar las demandas principales de sus usuarios (Fundación Biblioteca Virtual Miguel de Cer- vantes, 2008:3). Las principales conclusiones de la encuesta son:

- Ha sido algo mayor la participación de los hombres, el 59,2\%, en la encuesta.

- En relación a la edad de los usuarios, el mayor porcentaje, el 30\%, se encuentra en el intervalo entre 45 y 54 años. Y el $23,4 \%$ tienen de 55 a 64 años. Por lo tanto, el 53\% de los usuarios de la Biblioteca están comprendidos entre los 45 y 64 años. Los usuarios de menor edad son un porcentaje muy reducido: de 19 a 24 años sólo el 4,7\% y de 25 a 34 el 12,3\%. A pesar de la denominada brecha generacional, hay un $8,3 \%$ de usuarios con una edad comprendida entre los 65 y 74 años.

- España es el país de origen de la mayoría de sus usuarios, con el 46,6\%, seguido por Argentina, 12,5\% y México, $12,4 \%$.

- La novela es el género de las obras que se consultan más frecuentemente, por el 72\% y los artículos, el 71,6\%.

- Para el 82,8\% de los usuarios el formato de consulta preferente es el PDF, y el HTML para el 71,7\%. Sorprenden los reducidos porcentajes del audio, con el 32,8\%, del audiovisual con el 35,1\% y de multimedia con el 37,0\%. Aunque se especifica en el informe de la Fundación que ha aumentado el interés por este tipo de formatos, ya que en la encuesta realizada en el 2004, ninguno de ellos superaba el 30\%. 
- Un aspecto muy importante es que el $83 \%$ de los usuarios especifican que leen los contenidos en la pantalla de su ordenador. El $71 \%$ los guardan en su disco duro y el 56\% imprimen los contenidos.

- La mayoría de los usuarios, el 83,3\% afirman que leen íntegramente las obras que consulta en la biblioteca, y el $75,65 \%$ que consultan un fragmento.

- El 74,5\% de los usuarios utiliza la biblioteca virtual para el ocio, con fines profesionales el $61,9 \%$ y docentes o académico el $46,1 \%$.

A continuación se analizan los principales posicionamientos de la industria de los periódicos y de los lectores ante los periódicos online. Abadie, Maghiros y Pascu del Institute for Prospective Technological Studies, autores del informe "European Perspectivas on the Information Society: Annual Monitoring Synthesis and Emerging Trend Updates" especifican que los periódicos impresos son el subsector de la industria de contenidos que ha resultado más afectado por el desarrollo de Internet, lo que ha provocado unas caídas continuas de sus volúmenes de ventas (Abadie, Maghiros y Pascu, 2008:145). Por otra parte, se afirma que Internet ha "transformado las formas clásicas de trabajar en las redacciones de los diarios, ha modificado la relación consolidada durante décadas entre lectores y editores y ha abierto un periodo de incertidumbre en las empresas de comunicación” (Cerezo y Zafra, 2003:3). Además también señalan Cerezo y Zafra que la Red está haciendo que los medios busquen nuevas formulas para intentar rentabilizar los contenidos, y ha puesto en cuestión la credibilidad de las fuentes, el valor de la noticia impresa y el papel mediador del periodista.

A la industria de los periódicos se le presenta el problema de intentar rentabilizar las ediciones online. Se considera que las empresas de información han ofrecido sus productos gratis en Internet siguiendo "una estrategia empresarial bastante común, consistente en ofrecer un producto gratuitamente para más tarde obtener los ingresos a través de productos complementarios” (Pablo, Mañas y Cuadrado, 2006:68). El objetivo que perseguían las empresas de información era incrementar el número de sus usuarios y con ello conseguir más ingresos por publicidad (Brian, 1996:8), pero los ingresos obtenidos por los periódicos por publicidad son menores de los que se esperaban, y no consiguen compensar la reducción de los ingresos que tienen en las ediciones físicas. Además, se presenta el problema de que una vez que el usuario se ha acostumbrado a leer el contenido del periódico de una forma gratuita es muy difícil que pague por su lectura cuando la empresa periodística ha intentado cobrar por el acceso a sus contenidos online. También se señala que "bastantes ejemplos muestran que una vez que el consumidor se habitúa a no pagar por la adquisición de un determinado producto o servicio resulta 
difícil implantar un precio, especialmente si existen alternativas gratuitas" (Pablo, Mañas y Cuadrado, 2006:68). Esta realidad se ha presentando en el caso de los periódicos digitales españoles, que ha tenido una fuerte disminución de lectores en el momento que se ha intentado cobrar por sus contenidos.

Internet ha afectado tanto a la forma de trabajar de los medios, como a la propia información. En primer lugar los hábitos que tienen los consumidores de "lectura, acceso y búsqueda de la información han cambiado. Se han abierto las puertas de la información a todo el mundo... Los propios periodistas se han visto afectados dejando que la inmediatez prime sobre cualquier otro aspecto" (Cerezo y Zafra, 2003:3). Además, Internet facilita que el público pueda acceder directamente a las fuentes de la información sin necesidad de intermediarios ya que "por primera vez en la historia de la comunicación y gracias a Internet, las fuentes de información están alcance del público sin intermediarios profesionales. El periodista ya no es imprescindible, el sistema puede funcionar sin su participación directa (Cerezo y Zafra, 2003:5)

La generalización del uso de la Red ha provocado un profundo cambio en el modelo tradicional de los periódicos amenazando su situación igual que ha ocurrido con otros medios tradicionales "que requieren de fuertes inversiones para su puesta en marcha y mantenimiento, por lo que el poder de opinión e influencia se circunscribía a las grandes empresas editoriales o a los medios de carácter público" (Cerezo y Zafra, 2003:5). Pero con Internet se produce un profundo cambio de esta situación "el statu quo se transforma, la red satisface con creces las necesidades de inmediatez de la información, por encima de la radio y en dura competencia con la televisión; es un canal de distribución barato, para el que no existe limitaciones de espacio ni de tiempo" (Cerezo y Zafra, 2003:5). El creciente peso de Internet queda de manifiesto en los resultados del informe "Mediascope Europe 2007" que realiza la European Interactive Advertising Association (EIAA) donde se indica que las web más visitadas por los internautas españoles son las de noticias por el $80 \%$ y las de información local, por el 71\%. Estos resultados coinciden con los proporcionados por el informe de la Fundación BBVA "Segundo Estudio sobre Internet en España", en donde se especifica que el 82,0\% de los usuarios de Internet en España buscan información en Internet $\mathrm{y}$ leen noticias o periódicos online el 46,7\%.

Además, es distinto el proceso de lectura de las noticias en los periódicos físicos que en los periódicos online "los usuarios de los medios digitales navegan por Internet de información en información, ajenos, en la mayoría de los casos, a la afiliación o tendencia de las cabeceras de los medios tradicionales" (Cerezo y Zafra, 2003:8). Se considera que el lector de información en Internet no debe fidelidad a una cabecera 
concreta, sino que salta de un periódico a otro, ya que en la Red "la información está al alcance de un clic, deslocalizada y fragmentada”. Esta facilidad de acceso de la información plantea un problema a la industria periodística ya que "deben encontrar estrategia eficientes para fidelizar a los lectores, mantener la rentabilidad del negocio y el control sobre la información para preservar su influencia sobre los poderes económico y político" (Cerezo y Zafra, 2003:8).

Un problema que se presenta a la hora de intentar fidelizar a los lectores es la falta de diferenciación de las noticias en los distintos medios, ya que se afirma que "la información se realiza de una forma estándar y pierde capacidad de diferenciación. Es frecuente encontrar en Internet la misma noticia, con el mismo tratamiento en multitud de páginas web" (Cerezo y Zafra, 2003:5). A pesar de todos estos problemas se prevé un elevado crecimiento de los ingresos de los periódicos online, según el informe "Interactive content and convergence: Implications for the information society" la cifra de ingresos por contenidos digitales de los periódicos y revistas en la Unión Europea aumentarán desde 849 millones de euros en el año 2005 hasta los 2.001 en el 2010 (Screen Digest, Goldmedia y Rightscom, 2006:265). Este informe es el resultado de un estudio encargado por la Comisión Europea a las consultoras especializadas en medios de comunicación: Screen Digest, Goldmedia y Rightscom.
En España, el número de diarios digitales ha aumentado de forma continúa en los últimos años, en el año 2007 había 59 diarios digitales, Toro, Lozano y Milán especifican que este progresivo aumento "hace referencia a su consolidación paulatina como una nueva realidad periodística” (Toro, Lozano y Milán, 2008:152). Dentro de los países de la UE, España se coloca en el cuarto lugar en cuanto a número de cabeceras online detrás de Alemania, Italia y Suecia, por lo tanto la oferta existente en nuestro país es muy amplia. Desde el punto de vista de la demanda, la lectura de periódicos online es una costumbre que está plenamente consolidad entre los internautas españoles, según los resultados de " $10^{a}$ encuesta AIMC a usuarios de Internet" que realiza la Asociación para la Investigación de Medios de Comunicación (AIMC), el $67,2 \%$ de los encuestados afirman que han leído un periódico en su versión electrónica el día anterior a la realización de la encuesta de la AIMC. Estos resultados son muchos más elevados que los obtenidos por los otros medios online. El grado de acceso online reciente, suma de los accesos a los periódicos online ayer y anteayer, en relación a la fecha de la realización de la encuesta, casi triplica los valores obtenidos por la radio online, y casi quintuplica los accesos a la televisión online. Además, el periódico electrónico es el único medio online que tiene una frecuencia de uso mayor que la versión offline del medio. 
Los periódicos online, según la encuesta de la AIMC, han sido leídos en el día de ayer, por el $67,2 \%$ de los encuestados, y los periódicos físicos, por el 61,7\% (cuadro $\mathrm{n}^{\circ}$ 1). Si se incluye en la comparación también el acceso anteayer, se sigue manteniendo una mayor frecuencia de uso de la versión electrónica, ya que los resultados aumentan hasta el 71,2\% en la versión online y el $69,4 \%$ en papel. Sólo es superior el porcentaje de lectura de la versión impresa del periódico cuando se le suman a las respuestas anteriores el acceso en los últimos siete días, con un total de respuestas del $87 \%$, por el $82,1 \%$ en la versión electrónica.

Cuadro $n^{o} 1$. ¿Cuando ha sido la última vez que ha leído un periódico/diario?

\begin{tabular}{|l|l|l|}
\hline \multicolumn{2}{|c|}{$\begin{array}{l}\text { No electrónico } \\
\text { (papel) }\end{array}$} & \multicolumn{1}{|c|}{$\begin{array}{l}\text { Electrónico } \\
\text { (Internet) }\end{array}$} \\
\hline Ayer & 61,7 & 67,2 \\
\hline Anteayer & 7,7 & 4,0 \\
\hline $\begin{array}{l}\text { En los últimos } \\
7 \text { días }\end{array}$ & 17,6 & 10,9 \\
\hline $\begin{array}{l}\text { En los últimos } \\
\text { 30 días }\end{array}$ & 6,4 & 5,6 \\
\hline $\begin{array}{l}\text { Hace más } \\
\text { tiempo }\end{array}$ & & 5,3 \\
\hline $\begin{array}{l}\text { Nunca } \\
\text { Hace más } \\
\text { tiempo/Nunca }\end{array}$ & 5,4 & 4,4 \\
\hline NS/NC & 1,2 & 2,6 \\
\hline
\end{tabular}

Fuente: AIMC, Navegantes en la Red, $10^{a}$ encuesta AIMC a usuarios de Internet

Si se analizan otros informes, por ejemplo el "Segundo Estudio sobre Internet en España” de la Fundación BBVA, también se puede observar cómo ha aumentado en los últimos años el número de usuarios de
Internet que prefieren leer el periódico en la Red. El porcentaje ha crecido desde el $14,6 \%$ en el 2005 , hasta el $21,2 \%$, en el año 2008, y por lo tanto, se ha reducido el porcentaje de los internautas que prefieren los periódicos impresos desde el 75,7\% hasta el 63,4\%, respectivamente.

Según este informe de la Fundación BBVA la principal razón esgrimida por los encuestados para preferir leer la versión online de los periódicos estriba, para el 37,8\%, en la facilidad de encontrar la información que les interesa, el segundo motivo es la gratuidad del producto, para el 28,0\%. Otras razones mencionadas son: la facilidad para guardar las noticias que le interesan: 9,2\%, la información se actualiza constantemente: 7,5\%, mejor calidad de las imágenes: 6,7\% y que pueden leer el periódico en cualquier momento: 4,8\%. Por otra parte, los motivos de preferencia del periódico online son muy estables, ya que si se comparan los motivos mencionados en la encuesta de la Fundación BBVA del año 2008, con los esgrimidos en la encuesta del año 2005 que realizó la Fundación, se observa cómo han mantenido su grado de importancia a lo largo del tiempo. En la encuesta del año 2005, el principal motivo que mencionó el $61,1 \%$ de los internautas era la facilidad de encontrar la información y en segundo lugar, con un $18,4 \%$ que es un producto gratuito, seguido por la facilidad para guardar las noticias, para el 7,1\%. Se observa en esta comparación que el único motivo 


\section{Análisis comparativo de la tipología en España de los lectores de libros $y$ de periódicos en sus dos versiones offline $y$ online}

Este análisis se basa en la información que proporciona el informe "Hábitos de lectura y compra de libros en España 2007”, realizado por empresa Conecta Research \& Consulting para la Federación de Gremio de Editores de España (FGEE). Se comenta en la introducción del informe que uno de sus objetivos es la relación existente entre el consumo y utilización del libro con otros hábitos de consumo cultural, incluyéndose dentro de estos hábitos la lectura de periódicos. El informe se basa en los resultados obtenidos en 16.000 encuestas efectuadas durante el año 2007, según sus resultados el 56,9\% de la población de 14 o más años de edad son lectores de libros en España. Los lectores se desagregan en función de su frecuencia de lectura en dos grupos, en primer lugar están los lectores frecuentes que lo integran las personas que leen todos o casi todos los días $(25,5 \%)$ y los que tienen una frecuencia de lectura de una o dos veces por semana (15,5\%). El segundo grupo lo forman los lectores ocasionales, que son los que leen al menos una vez al mes $(10,5 \%)$ o alguna vez al trimestre (5,4\%) (FGEE, 2008: 18).
Por su parte, el 77,4\% de la población de 14 o más años de edad leen periódicos. La mayor frecuencia de lectura, con el 54,9\% de los lectores, es la frecuencia de 5 a 7 días a la semana, seguida por el 24,9\% que los leen de 1 a 2 días a la semana y el $12,8 \%$ con una frecuencia de 3 a 4 días. Con un nivel de lectura de alguna vez al mes o menor, los leen el 7,4\%. Los periódicos con un mayor número de lectores son los de información general, leídos por el $85,8 \%$, a continuación se encuentran los deportivos y los gratuitos, con unos porcentajes del $19,9 \%$ y del 15,7\%, respectivamente, en último lugar se sitúan los económicos, que sólo los leen el 5,0\% de los lectores de periódicos (FGEE, 2008: 129).

A continuación se va a realizar un análisis comparando la tipología de los lectores de libros físicos con la que tienen los lectores de ebooks, el análisis de la población lectora de libros impresos se realiza considerando las características de la población que son lectores frecuentes. No se puede realizar la comparación con los datos del total de población lectora, porque en el informe 
"Hábitos de lectura y compra de libros del año 2007”, utilizado como fuente de la información, no hay datos sobre ella. Siguiendo este criterio se excluyen de este análisis a los lectores que leen con una frecuencia de alguna vez al mes, que constituyen el 10,5\%, de la población, y a los que leen alguna vez al trimestre, que suponen el 5,4\%.

La población lectora frecuente de libros impresos está formada, como se ha comentado anteriormente, por las personas que tienen una frecuencia de lectura de todos o casi todos los días, más los lectores que leen de una o dos veces por semana. Este colectivo es 2,4 veces mayor que los lectores de ebooks. Este dato refleja la reducida implantación de los libros electrónicos en España (cuadro $n^{\circ} 2$ ). Se producen algunas diferencias en la tipología de los lectores de libros en sus dos formatos en cuanto al género, en los lectores de ebooks es superior el porcentaje de lectores hombres, el $19,4 \%$ de la población masculina, que el porcentaje de lectores mujeres, el 14,0\%, por lo tanto, hay una diferencia de 5,4 puntos porcentuales. En cambio en el caso de los libros impresos, es al revés, el porcentaje de lectoras es 7,2 puntos porcentuales mayor.
Cuadro $n^{o}$ 2. Principales características de los lectores (en porcentajes)

\begin{tabular}{|l|l|l|l|l|}
\hline \multicolumn{2}{|c|}{$\begin{array}{l}\text { Lectores } \\
\text { frecuen- } \\
\text { tes libros }\end{array}$} & \multicolumn{1}{l}{$\begin{array}{l}\text { Leer/ } \\
\text { descar- } \\
\text { gar } \\
\text { ebooks }\end{array}$} & \multicolumn{1}{l|}{$\begin{array}{l}\text { Lectores } \\
\text { prensa } \\
\text { offline }\end{array}$} & $\begin{array}{l}\text { Lectura } \\
\text { online } \\
\text { periódi- } \\
\text { cos }\end{array}$ \\
\hline Total & 41,0 & 17,1 & 77,4 & 54,7 \\
\hline Sexo & & & & \\
\hline Hombre & 37,4 & 19,4 & 82,7 & 61,4 \\
\hline Mujer & 44,6 & 14,0 & 72,1 & 45,7 \\
\hline Edad & & & & \\
\hline De 14 a 24 & 50,7 & 21,2 & 66,7 & 51,7 \\
\hline De 25 a 34 & 47,2 & 19,0 & 79,4 & 62,9 \\
\hline De 35 a 44 & 44,7 & 13,9 & 81,8 & 52,0 \\
\hline De 45 a 54 & 44,3 & 13,6 & 83,2 & 49,6 \\
\hline De 55 a 64 & 37,6 & 9,8 & 79,3 & 47,0 \\
\hline $\begin{array}{l}\text { 65 años y } \\
\text { más }\end{array}$ & 22,4 & 19,5 & 71,9 & 53,4 \\
\hline
\end{tabular}

Fuente: Federación de Gremios de Editores de España, Hábitos de lectura y compra de libros en España 2007

También se producen algunas disparidades en cuanto a la edad de los lectores. En ambos casos el principal grupo de lectores son las personas más jóvenes, los que tienen de 14 a 24 años. Pero en el caso de los libros electrónicos, el segundo grupo con un mayor número de lectores lo forman las personas de 65 y más años de edad, colectivo que tiene el índice más bajo de lectores en libros físicos. Además, en su caso es muy reducida la diferencia existente entre los porcentajes de lectores de ebooks y libros impresos, sólo son 2,9 puntos porcentuales, cuando en todas las demás edades esta diferencia está alrededor de los 30 puntos porcentuales. 
Excluido el grupo de lectores de más edad, el porcentaje de lectores de ebooks se reduce a medida que aumenta la edad, siendo el colectivo con un menor número de lectores el formado por los individuos entre 55 y 64 años. En el caso de los libros impresos, también se reduce el porcentaje de lectores a medida que aumenta la edad, correspondiendo el porcentaje más bajo a las personas que tienen mayor edad, los de 65 y más años (cuadro n ${ }^{\circ} 2$ ).

Por otra parte, es sorprendente el reducido nivel de lectores de ebooks entre la población más joven, tanto por su nivel de lectura de libros impresos, como su mayor incorporación a la Sociedad de la Información, haría suponer a priori, un mayor número de lectores de libros electrónicos.

Por otra parte, en ambos tipos de soporte de libro, aumenta el número de lectores a medida que estos tienen un mayor el nivel de estudios, pero en lo referente a los libros físicos se produce una mayor diferencia entre los distintos niveles de formación, concretamente el porcentaje de lectores universitarios es 3,1 veces mayor que el porcentaje de los sólo tienen una formación hasta primaria (cuadro $n^{\circ} 3$ ). Por el contrario, en el caso de los ebooks, se reducen estas diferencias, y el porcentaje de lectores con formación universitaria es un $50 \%$ mayor que el correspondiente a los lectores con un menor nivel de formación. De estos datos se deduce un bajo grado de utilización de los libros electrónicos, en términos relativos, por parte de los lectores de mayor formación.

Cuadro $n^{o}$ 3. Principales características de los lectores (en porcentajes)

\begin{tabular}{|l|l|l|l|l|}
\hline \multicolumn{2}{|c|}{$\begin{array}{l}\text { Lectores } \\
\text { frecuen- } \\
\text { tes libros }\end{array}$} & $\begin{array}{l}\text { Leer/ } \\
\text { des- } \\
\text { cargar } \\
\text { ebooks }\end{array}$ & $\begin{array}{l}\text { Lecto- } \\
\text { res } \\
\text { prensa } \\
\text { offline }\end{array}$ & $\begin{array}{l}\text { Lectura } \\
\text { online } \\
\text { periódi- } \\
\text { cos }\end{array}$ \\
\hline Estudios & & 13,0 & 72,9 & 41,5 \\
\hline Hasta primaria & 22,2 & 16,3 & 79,4 & 49,4 \\
\hline Secundarios & 46,5 & 19,4 & 85,1 & 63,9 \\
\hline universitarios & 68,9 & & & \\
\hline $\begin{array}{l}\text { Situación } \\
\text { Laboral }\end{array}$ & & 14,7 & & 56,4 \\
\hline Ocupados & 45,6 & 23,4 & & 51,2 \\
\hline Estudiantes & 54,4 & 13,0 & & 27,6 \\
\hline Amas de casa & 32,0 & 16,4 & & 63,8 \\
\hline Jubilados & 25,2 & 20,6 & & 53,1 \\
\hline Parados & 39,6 & & \\
\hline
\end{tabular}

Fuente: Federación de Gremios de Editores de España, Hábitos de lectura y compra de libros en España 2007

En relación a la situación laboral del lector, tanto en los libros impresos como en los ebooks, el mayor porcentaje de lectores se produce entre los estudiantes. Pero en relación a los libros físicos el segundo colectivo son los ocupados, y en los ebooks, los parados. Por otra parte, los jubilados es el colectivo que tiene un menor nivel de lectores en el caso de los libros impresos, en cambio en el caso de los libros electrónicos son el tercer grupo en importancia por número de lectores. Además, en los jubilados la diferencia entre número de lectores de libros impresos y de ebooks es únicamente de 8,8 puntos porcentuales, esta diferencia es menos de la mitad de la que se produce en el caso de las amas de 
casa y de los parados, y es una tercera parte de la que hay en los colectivos de ocupados y estudiantes. Curiosamente en este último colectivo es donde se manifiesta la mayor diferencia entre los porcentajes de lectores, 31 puntos porcentuales. Inicialmente se podría suponer que debido tanto a su mayor uso de Internet, como a su elevado porcentaje de lectura de libros impresos, habría un mayor número de lectores de ebooks.

Por otra parte, hay una relación directa entre el tamaño del municipio y el porcentaje de población lectora en ambos formatos de libros. Pero en el caso de los ebooks se reducen las diferencias en cuanto a porcentajes de lectores entre los distintos tamaños de municipio. En los libros impresos hay una diferencia de 10,2 puntos porcentuales entre los municipios de más de 1 millón de habitantes, que son los municipios con un mayor porcentaje de lectores y los municipios de 200.001 a medio millón, los municipios que ocupan el segundo lugar, pero en los libros electrónicos la diferencia es de sólo 0,4 puntos porcentuales (cuadro $\mathrm{n}^{\circ} 4$ ).

También se pueden observar cómo se reduce la brecha entre el número de lectores según el tamaño de residencia del lector. Si se comparan los valores extremos de la distribución de porcentaje de lectores por tamaño del municipio, en el

\begin{tabular}{|c|c|c|c|c|}
\hline & $\begin{array}{l}\text { Lectores } \\
\text { frecuen- } \\
\text { tes libros }\end{array}$ & $\begin{array}{l}\text { Leer/ } \\
\text { descar- } \\
\text { gar } \\
\text { ebooks }\end{array}$ & $\begin{array}{l}\text { Lectores } \\
\text { prensa } \\
\text { offline }\end{array}$ & $\begin{array}{l}\text { Lectura } \\
\text { online } \\
\text { periódi- } \\
\text { cos }\end{array}$ \\
\hline \multicolumn{5}{|l|}{$\begin{array}{l}\text { Tamaño muni- } \\
\text { cipio (en 1.000) }\end{array}$} \\
\hline $\begin{array}{l}\text { Menos de } 10 \\
\text { hab. }\end{array}$ & 32,5 & 14,6 & 71,0 & 49,1 \\
\hline $\begin{array}{l}\text { De } 10.001 \text { a } \\
50.000 \text { hab. }\end{array}$ & 37,9 & 14,6 & 75,5 & 54,6 \\
\hline $\begin{array}{l}\text { De } 50.001 \text { a } \\
200.000 \text { hab. }\end{array}$ & 43,2 & 17,2 & 79,6 & 54,0 \\
\hline $\begin{array}{l}\text { De } 200.001 \text { a } \\
500.000\end{array}$ & 45,2 & 20,8 & 82,3 & 54,2 \\
\hline $\begin{array}{l}\text { De } 500.001 \mathrm{a} \\
1.000 .000 \mathrm{hab} .\end{array}$ & 45,0 & 17,6 & 78,3 & 51,2 \\
\hline $\begin{array}{l}\text { Más de } \\
1.000 .0001 \text { hab. }\end{array}$ & 55,4 & 21,2 & 85,4 & 64,1 \\
\hline
\end{tabular}
caso de los libros impresos, el porcentaje de población lectora en los municipios de menor tamaño, los que tienen menos de
10.000 habitantes, supone el 42\% del porcentaje de población lectora en los municipios de más de un millón de habitantes. En cambio, en el caso de los ebooks se reduce al 31,13\%. Por lo tanto, se puede afirmar que se produce un uso más intensivo de los libros electrónicos, en términos relativos, en los municipios más pequeños, es decir, que la disponibilidad de los libros electrónicos facilita a los residentes en estos municipios el acceso a una oferta de productos que no están fácilmente disponibles en libros físicos, y por ello, se produce una mayor utilización de los ebooks. Claramente en estos casos los ebooks facilitan que estas poblaciones puedan acceder a leer unas obras que si no existiesen los libros electrónicos les sería mucho más difícil a los lectores interesados poder leer.

\section{Cuadro $n^{\circ}$ 4. Principales características de los lectores (en porcentajes)}


A continuación se analizan las principales diferencias que surgen en la tipología de los lectores de periódicos impresos y los online, aunque en este caso las diferencias son menos acusadas que las que se producen entre los lectores de libros impresos y de ebooks. El número de lectores de periódicos físicos es 1,4 veces mayor que el que tienen los lectores de periódicos online. Y en ambos soportes es mayor el número de lectores masculinos que femeninos, en los periódicos impresos el porcentaje de lectoras es un $12,82 \%$ menor, pero en los online se agudizan las diferencias de género con un porcentaje de lectoras un 25,57\% menor.

En las versiones físicas los principales colectivos de lectores, están constituidos por los individuos de mediana edad. El primer grupo lo integran las personas con una edad comprendida entre 45 a 54 años, con el $83,2 \%$ de lectores, y el segundo, los que tienen entre 35 a 44 , con el $81,8 \%$. A continuación se encuentran con el 79,4\% de lectores de periódicos las personas entre 25 a 34 años y en cuarto lugar con el $79,3 \%$ los de 55 a 64 años. Sin embargo, en las versiones online se produce un cambio sustancial en la estructura de los lectores según su edad, y adquieren una mayor importancia colectivos que tienen un bajo porcentaje de lectores de periódicos físicos. El grupo más importante lo integran con el $62,9 \%$ de lectores las personas que tienen una edad entre 25 a 34 años, seguidos por los de 65 y más años, con el 53,4\% de lectores, en la versión física de los periódicos estos colectivos ocupan el tercer y quinto lugar, respectivamente. Los individuos más jóvenes, de 14 a 24 años, son el cuarto grupo en los periódicos online, con el 51,7\% de lectores, y como en el caso de los colectivos anteriores también aumentan su peso con respecto al que tienen en las versiones físicas, en donde ocupan el último lugar. En cambio las personas con una edad entre 45 y 54 años, que son el primer colectivo de lectores en los periódicos impresos, en la versión online ocupan el penúltimo lugar con el 49,6\% (cuadro $\mathrm{n}^{\circ}$ 1).

En los dos soportes de periódicos, aumenta el porcentaje de lectores a medida que se eleva el nivel de formación del lector. En el caso de la prensa online se agudizan las diferencias existentes entre los distintos niveles de formación, el porcentaje de lectores universitarios es un 50\% superior al que tienen los lectores con formación primaria. En cambio, en el caso de los periódicos impresos, es solamente un 15\% mayor (cuadro $n^{\circ} 2$ ). También en las dos versiones, hay una relación directa entre el porcentaje de la población lectora y el tamaño del municipio de residencia, en el caso de los periódicos online el porcentaje de lectores de los municipios de más de un millón de habitantes es un 15\% superior al que existe en los municipios más pequeños, los que tienen menos de 10.000 habitantes. Pero en el caso de los periódicos impresos esta diferencia aumenta hasta el 20\%. Por 
lo tanto, Internet facilita el acceso a los periódicos de los individuos que tienen una menor oferta de periódicos impresos, los que residen en los municipios más pequeños (cuadro $n^{\circ} 3$ ). Las nuevas tecnologías producen el mismo efecto en los periódicos, que el ya comentado en el caso de los ebooks, facilitando una mayor demanda de ambos productos.

La comparación de los principales datos de los lectores de ebooks con los que tienen los lectores de periódicos online, permite apreciar de forma inmediata la mayor consolidación en el mercado español de los periódicos online, con un porcentaje de lectores del 54,7\% de la población de 14 o más años de edad. En cambio, en el caso de los ebooks, el porcentaje de lectores se reducen hasta el $17,1 \%$. También se aprecia este mayor desarrollo si se compara el grado de implantación de las versiones online de los periódicos y libros con el grado de uso de las versiones offline, el número de lectores de periódicos físicos es sólo 1,4 veces mayor que el de lectores de sus versiones online. Sin embargo, el porcentaje de lectores de libros impresos es 2,4 veces mayor que el de lectores de ebooks.

Desde el punto de vista del género del lector, es mayor el número de lectores masculinos de las versiones digitales, agudizándose la brecha de género con el acceso a este tipo de versiones. En el caso de los periódicos impresos el porcentaje de lectoras es un $12,83 \%$ menor respecto al de los hombres y la diferencia se eleva hasta el $25,57 \%$ en el caso de los periódicos online. Por su parte, en el caso de los libros la diferencia existente es todavía mucho mayor: en los libros impresos el porcentaje de lectoras es mayor que el de lectores, concretamente 7,2 puntos porcentuales más. Por el contrario, en los ebooks, ocurre lo contrario, es mayor el porcentaje de lectores, con una diferencia a su favor de 5,4 puntos porcentuales, es decir, el porcentaje de lectoras es un 27,83\% menor. Por lo tanto, las diferencias de género existentes en el acceso digital a periódicos y libros son muy parecidas.

Si se considera la variable edad del lector, las versiones online alteran el peso de los grupos de lectores, especialmente en el caso de los periódicos. En ambos casos, tanto en ebooks como en los periódicos online sorprende el aumento de peso, en términos relativos, de la población de más edad, de las personas que tienen 65 y más años, que son el segundo colectivo con un mayor número de lectores en ambas versiones online. En cambio, en las versiones offline son el último grupo en el caso de los libros y el penúltimo en los periódicos.

Asimismo sorprende el reducido peso de los individuos más jóvenes, de edad comprendida entre 14 y 24 años. Su mayor uso de las nuevas tecnologías, en comparación con los otros colectivos edad superior, harían pensar una mayor utilización de las versiones online de los libros y periódicos, especialmente en el caso de los libros, en 
los que tienen unos elevados índices de lectura en la versión offline. En relación a los periódicos online son el cuarto grupo en cuanto a número de lectores, pero debe tenerse en cuenta que son el último en las versiones offline de los mismos. En cambio, si se produce un mayor grado de acceso a los periódicos online, en términos relativos, en los individuos con edades entre 25 a 34 años de edad que son su primer grupo lector, aunque el tercero, en el caso de los periódicos impresos. Por el contrario, en el caso de los libros, son el segundo colectivo en las ediciones impresas y pasan al tercero en los ebooks.

Por otra parte, el grado de lectura de los libros disminuye con la edad del lector, tanto de los libros impresos, como de los ebooks, con la excepción de los individuos de más de 65 años, ya indicado. En cuanto a los periódicos los cambios que se produce entre las dos versiones son más elevados, como ya se comentó anteriormente, pasando el primer grupo de lectores de periódicos físicos, el formado por los individuos de 45 a 54 años, a ocupar la quinta posición en los online. En cambio, se produce un acercamiento a este medio, en su versión online, de las personas que tienen entre 25 y 43 años. Por lo tanto, la versión online de los periódicos produce un mayor acercamiento a este medio, en términos relativos, de los individuos de menos de 34 años, un cierto alejamiento de los que tienen entre 35 y 64 años, y un consumo mucho más elevado de las personas mayo- res de 65 años. En la medida que generalice el uso de los ebooks en España, sería previsible un cambio parecido en la estructura de edad de sus consumidores. De hecho, en lo referente a los individuos de más de 65 años, ya se ha producido ese cambio.

El número de lectores de periódicos aumenta con el grado de formación del lector, independientemente de que sea la versión online o la offline. Pero como se ha analizado con anterioridad, en la versión online se agudizan las diferencias existentes, ya que el porcentaje de lectores en individuos con formación universitaria es un $50 \%$ superior al porcentaje con formación primaria, siendo solo un 15\% en el caso de los periódicos impresos. En los ebooks ocurre lo contrario: el porcentaje de lectores en las personas con formación universitaria es un 49,2\% superior que en formación primaria, pero en los libros impresos el porcentaje de lectores es 3,1 veces mayor.

También existe una relación de tipo positivo entre el número de lectores de periódicos y de los libros en sus dos versiones y el tamaño del municipio donde viven. Pero el acceso a las versiones online disminuye las diferencias existentes en las offline entre los porcentajes de lectores de los municipios más grandes y los de menor tamaño. En el caso de los periódicos impresos, el porcentaje de lectores que tienen los municipios de mayor tamaño es un 20\% superior y se reduce hasta el 15\% en los periódicos online. En el caso de los libros impre- 
sos, este porcentaje es del $42 \%$, y disminuye hasta el $31,13 \%$ con los ebooks. Por lo tanto, en ambos casos se reduce en un $25 \%$, lo que señala el papel que tiene Internet permitiendo acceder a las personas que viven en los municipios de menor tamaño a los productos culturales online, aumentando la oferta de que disponen, y eliminado las restricciones y limitaciones existentes en sus mercados offline de periódicos y libros impresos.

\section{Conclusiones}

- El creciente nivel de penetración de la banda ancha está provocando que la compañías de medios diversifiquen los canales de distribución de sus contenidos guiándose por la premisa de "anytime, anywhere" para de esta forma poder satisfacer las necesidades de sus clientes. Pero la migración hacia los formatos digitales está afectando de forma negativa a los ingresos obtenidos por los formatos físicos.

- La banda ancha permite nuevas posibilidades de ocio y entretenimiento, las nuevas ventanas implican nuevos modelos de negocio. Sin embargo se está produciendo una fragmentación de las audiencias motivada por los cambios en los hábitos de los consumidores que reparten su atención entre las ventanas clásicas de distribución y las nuevas.

- En el año 2007 la distribución de contenidos digitales sólo representó el 5\% de los ingresos mundiales del sector de ocio y medios de comunicación, pero estos contenidos representarán el $11 \%$ de la facturación total del sector en el año 2012.
- En el mercado español se estima que la edición de libros crecerá en el periodo de 2008 a 2012 a una media anual del $2,1 \%$, y la edición de periódicos del $2,9 \%$. Siendo unos de los subsectores que tendrán un crecimiento más reducido, la edición de libros crecerá en el periodo a una tasa que es el $25 \%$ de la tasa media del sector de ocio y medios de comunicación en España, y la tasa de la edición de periódicos es el 37\%.

- Se prevé que los ebooks tendrán un elevado crecimiento a nivel mundial, hasta llegar a suponer el 5,2\% del total de ingresos de la industria editorial en el 2012.

- No se prevé que los ebooks canibalicen la venta de libros físicos, debido a que su impresión por parte del lector no es eficiente por los costes de papel y tinta, y a las reticencias de muchos lectores a la lectura de los textos en pantalla. La aparición en el mercado de nuevos dispositivos para la lectura de ebooks, como el Kindle de Amazon y el Reader de Sony han supuesto una auténtica revolución. 
- El mercado español de la edición de periódicos se estima que crecerá a una media anual del 2,9\% en el periodo 2008-2012, que es una tasa superior a la media mundial estimada, 2,2\%.

- Los ingresos de los periódicos digitales se estima que llegarán a representar en el año 2012 el 10\% del total de ingresos del mercado de los periódicos a nivel mundial.

- La migración de los lectores de las ediciones en papel a las online provocará un incremento de la publicidad online, pero al mismo tiempo reducirá los volúmenes de circulación de los periódicos físicos. Por lo tanto, se producirá una canibalización de las versiones impresas por la online.

- El uso masivo de Internet está provocando grandes cambios tanto en las empresas de contenidos, como en las formas de consumo de dichos contenidos. Estos cambios están provocando la potenciación de una economía interconectada que se basa en la información. Además, se está produciendo un nuevo tipo de relación entre los clientes, los productos y el canal de distribución.

- El principal efecto que provocan las nuevas tecnologías en las empresas se debe a las externalidades de red.

- Internet afecta a la empresa tanto desde el punto de vista de la demanda como de los costes y provoca una profunda transformación de los mercados, espe- cialmente en el sector de entretenimiento donde el tratamiento de la información y de los contenidos es clave. Asimismo sufren transformaciones los sectores de las telecomunicaciones y de las infraestructuras (sectores de hardware, software y electrónica). Todos estos sectores están inmersos en un proceso de convergencia.

- Internet ha implicado una profunda transformación del modelo de negocio de las siguientes industrias: medios de comunicación, música, cine, industria editorial e intermediarios financieros.

- Se considera el consumo de los bienes culturales y de entretenimiento es el sector que está siendo más afectado por el desarrollo de la Sociedad de la Información

- Las nuevas tecnologías reducen la asimetría de información existente entre los consumidores y los productores, permitiendo que los primeros obtengan de forma fácil información relacionada con los distintos precios y calidades de los productos disponibles.

Un hecho muy relevante que está ocurriendo en los mercados de los bienes culturales y de entretenimiento es que, la inmediatez de la información, la mejora de la calidad de las comunicaciones y los cambios en las forma de entretenimientos facilitados todos ellos por una mayor implantación de las TIC en los hogares no están implicando un au- 
mento del gasto de las familias en dichos bienes.

- En la actualidad gracias a la convergencia tecnológica, los servicios de comunicación, información y entretenimientos son ofrecidos a los consumidores por diversas empresas, no sólo por las que antes los suministraban de forma habitual, sino que también han aparecido otras nuevas que los suministran y que compiten con las que tradicionalmente los ofrecían, lo que ha implicado un aumento de la oferta de servicios para los consumidores y una mejora en su capacidad de negociación con dichas empresas.

- En los bienes y servicios culturales ligados a la Sociedad de la Información se están produciendo grandes cambios tanto en relación a las cantidades consumidas de los mismos, como en la forma de utilización de dichos productos. Además su consumo en unidades físicas está aumentado por la continúa reducción de sus costes medios.

- El mayor uso de las TIC en el hogar ha permitido a las familias un consumo gratuito de bienes culturales y de entretenimiento, ya sea porque estos se proporcionan gratis a través de Internet, o por los sistemas de intercambio de ficheros.

- Las empresas productoras de bienes de información online incurren en unos elevados costes fijos al producir el pri- mer ejemplar del bien, pero como sus costes variables son muy pequeños, el coste medio se reduce cuanto mayor sea su volumen de producción. Una característica que tienen los bienes de información es que su producción tiene unos costes fijos muy altos, pero también tiene unos costes marginales muy reducidos.

Se considera que las muestras de productos de información son eficaces para incrementar el mercado ya que al ser lo que se denomina en la teoría económica bienes de experiencia, la muestra permite que el consumidor pueda conocerlos. Por otra parte, debido a que tiene un coste marginal cero, o casi cero, le cuesta muy poco a la empresa proporcionar estas muestras

- Existe una profunda divergencia entre las opiniones de los empresarios del sector editorial europeos y los españoles en relación a cual será su principal fuente de ingresos dentro de 10 años. Según los resultados de una encuesta realizada por la organización de la Feria de Frankfurt del año 2008 a 1.000 profesionales del sector, el $40 \%$ consideran que la cifra de negocio de los ebooks superará a la de los libros en papel dentro de 10 años. Por el contrario, para el $48 \%$ de los profesionales españoles encuestados en LIBER 2008, los libros en papel continuarán siendo la principal fuente en el 2020, y sólo el 16\% opinan 
que los libros electrónicos serán la primera fuente de ingresos.

- Es algo mayor el número de estudiantes universitarios que utilizan ebooks para los trabajos de clase o investigaciones, frente a los libros impresos. Además, los recursos considerados como más fiables por parte de los estudiantes son estos últimos y los ebooks. A continuación se encuentran los libros de texto impresos, los diccionarios y enciclopedias en papel, los periódicos físicos, y posteriormente los periódicos online, los libros de texto online, y los diccionarios y enciclopedias online.

- Se afirma que los periódicos impresos constituyen el subsector de la industria de contenidos que ha resultado más afectado por el desarrollo de Internet, provocando unas caídas continuas de sus volúmenes de ventas. Un problema que se presenta a la industria de los periódicos es intentar rentabilizar sus ediciones online.

- Se estima que la cifra de ingresos por contenidos digitales de los periódicos y revistas en la Unión Europea aumentarán desde 849 millones de euros en el año 2005 hasta los 2.001 en el 2010 .

- El periódico electrónico es el único medio online que tiene una frecuencia de uso por parte de los internautas mayor que la versión offline del medio.

- Las principales ventajas de los periódicos online según sus lectores son: la fa- cilidad de encontrar la información que les interesa y que es un producto gratuito. Otras ventajas son: la facilidad para guardar las noticias, la información se actualiza constantemente, mejor calidad de las imágenes y que pueden leer el periódico en cualquier momento.

- La comparación de los principales datos de los lectores de ebooks con los lectores de periódicos online, permite apreciar de forma inmediata la mayor consolidación en el mercado español de los periódicos online, con un porcentaje de lectores del $54,7 \%$ de la población de 14 o más años de edad, en cambio en el caso de los ebooks su porcentaje de lectores se reducen hasta el $17,1 \%$.

- Desde el punto de vista del género del lector, es mayor el número de lectores masculinos en las versiones digitales, agudizándose la brecha de género con el acceso a las versiones electrónicas de los periódicos y de los libros.

- Si se considera la variable edad del lector, las versiones online alteran el peso de los grupos de lectores de las versiones físicas, especialmente en el caso de los periódicos online

- El número de lectores de periódicos aumenta con el grado de formación del lector, independientemente de que sea la versión online o la offline. En la versión online se agudizan las diferencias existentes, ya que el porcentaje de lectores en individuos con formación uni- 
versitaria es un $50 \%$ superior al porcentaje con formación primaria, siendo solo un $15 \%$ en el caso de los periódicos impresos. En los ebooks ocurre lo contrario, el porcentaje de lectores en las personas con formación universitaria es un $49,2 \%$ superior que en formación primaria, pero en los libros impresos el porcentaje de lectores es 3,1 veces mayor.

- Existe también una relación de tipo positivo entre número de lectores de periódicos y de los libros, en sus dos versiones, y el tamaño del municipio donde viven. Pero el acceso a las versiones online disminuye las diferencias que se producen en las versiones offline entre el porcentaje de lectores de los municipios más grandes y los de menor tamaño. Por lo tanto, Internet permite acceder a las personas que viven en los municipios de menor tamaño a productos culturales online, aumentando la oferta de productos de que disponen, y eliminado las restricciones y limitaciones existentes en sus mercados offline de periódicos y libros impresos.

\section{Referencias}

ABADIE, F., MAGHIROS, I. Y PASCU, C. (Editors) (2008). European Persèctives on the Information Society: Annual Monitoring Síntesis and Emerging Trend Updates. Luxembourg: Institute for Prospective Technological Studies, Joint Research Centre, European Commission. Office for Official Publications of the European Communities.

AIMC (ASOCIACIÓN PARA LA INVESTIGACIÓN DE MEDIOS DE COMUNICACIÓN) (2008). Navegantes en la Red. $10^{\circ}$ encuesta AIMC a usuarios de Internet. Madrid: AIMC.

BRIAN, A. (1996). Increasing Returns and the New World of Business. Harvard Business Review, JulioAgosto.

CASALS CARRO, M.J. (2006). La enseñanza del periodismo y las nuevas tecnologías de la información y de la comunicación, Estudios sobre el mensaje periodístico, 12, 59-70.

CASALS CARRO, M.J. (2005). Otro mundo es posible: de la seudociencia de la comunicación a la defensa del periodismo como sentido de la realidad. Revista Anthropos, 209, 91-118.
CASSIMAN, B. y SIEBER, S. (2001). El impacto de Internet sobre la estructura de los mercados. Economía Industrial, 339, 13-24.

CEREZO, J.M. y ZAFRA, J.M. (2003). El impacto de internet en la prensa. Cuadernos/ Sociedad de la Información, 3. Madrid: Fundación Auna.

DOSDOCE.COM y EDICIONA (2008).

Digitalización del libro en España, en

http: / / www.dosdoce.com (consulta: 1 de abril de 2009)

EBRARY (2008). 2008 Global Student E-book Survey. Palo Alto (USA): Ebrary.

ECONOMIDES, N. (2005). The Incentive for Vertical Integration. The Networks, Electronic Commerce and Telecommunications Institute, Working Paper, 05-01, January

EUROPEAN INTERACTIVE ADVERTISING ASSOCIATION (EIAA) (2007). Mediascope Europe 2007. Londres: European Interactive Advertising Association.

FARIAS, P. y ROSES, S. (2009). La crisis acelera el cambio del negocio informativo. Estudios sobre el Mensaje Periodístico, 15, 15-32 
FEDERACIÓN DE GREMIOS DE EDITORES DE ESPAÑA (2008). Hábitos de lectura y compra de libros 2007. Madrid: Ministerio de Cultura y Federación de Gremios de Editores de España.

FOWLER, T.B. (2001). The Impact of Technology on Intellectual Property Rights. The

Telecommunications Review, 5, 33-49

FRIBERG, R., GANSLANDT, M. Y

SANDSTRÖM, M. (2001). Pricing Strategies in ECommerce: Bricks vs. Clicks. Research Institute of Industrial Economics. Working Paper Series 559

FUENTELSAZ, L., MAICAS, J.P. y POLO, Y. (2002). La generación de valor en la Economía Digital, V Encuentro de Economía Aplicada http: / / www.revecap.com/encuentros/anteriores/ veea/autores/F/fuentelsaz.html (consulta: $11 \mathrm{de}$ mayo de 2009)

FUNDACIÓN BBVA. (2008). Segundo Estudio sobre Internet en España. Madrid: Fundación BBVA, Departamento de Estudios Sociales.

FUNDACIÓN BBVA. (2005). Estudio sobre Internet en España. Madrid: Fundación BBVA, Departamento de Estudios Sociales.

FUNDACIÓN BIBLIOTECA VIRTUAL MIGUEL DE CERVANTES. (2008) Encuesta a usuarios de la Biblioteca Virtual Miguel de Cervantes sobre hábitos de lectura. Madrid: Fundación Biblioteca Virtual Miguel de Cervantes.

HARBAUGH, R y KHEMKA, R. (2001) Does Copyright Enforcement Encourage Piracy?. Claremont Colleges Working Papers, Agosto.

LARRAÑAGA, J. (2009). Características de la demanda de periódicos online y ebooks como recursos para la formación online de investigadores en Documentación en RAMOS; G.P., LÓPEZ, M., GUTIÉRREZ, A. y LARRAÑAGA, J. (Editores). Aplicaciones tecnológicas en la formación de investigadores en el campus virtual. 928. San Luis Potosí (México): Escuela de Ciencias de la Información, Universidad Autónoma de San Luis Potosí.

LÓPEZ SÁNCHEZ, J.I. y ARROYO BARRIGÜETE, J.L. (2006). Externalidades de red en la economía digital: una revisión teórica. Economía Industrial, 361, 21-32.
LÓPEZ SUÁREZ, M. (2008) Literatura y medios de comunicación. Madrid: Ediciones del Laberinto.

PABLO MARTÍ, F., MAÑAS ALCÓN, E. y CUADRADO ROURA, J.R. (2006). El impacto de las TIC en las formas de consumo familiar. Economía Industrial, 360, 61-75.

PORTER, M.E.(2001). Strategy and the Internet. Harvard Business Review, Marzo, 63-78.

PRICEWATERHOUSECOOPERS (PwC) (2008a). Global Entertainment and Media Outlook: 2008-2012.

Madrid: PricewaterhouseCoopers.

PRICEWATERHOUSECOOPERS (PwC) (2008b). Global Entertainment and Media Outlook: 2008-2012.

Nota de Prensa. Madrid: PricewaterhouseCoopers.

SCREEN DIGEST, GOLDMEDIA y RIGHTSCOM. (2006). Interactive content and convergence: Implications for the information society. A Study for the European Commission (DG Information Society and Media). London: Screen Digest Limited.

SHAPIRO, C. y VARIAN, H. (2000). El dominio de la Información. Una guía estratégica para la economía de la Red, Barcelona, Antoni Bosch.

TORO MARTÍN de J.M., LOZANO MUÑOZ, V. y MILÁN LÓPEZ, M., 2008, "La prensa digital" en BEL MALLÉN, J. I. et al (coord): Libro Blanco de la prensa diaria. 2009. 152-167. Madrid: Asociación de Editores de Diarios Españoles (AEDE).

VALOR, J. y HESS, M. (2001). Portales, proveedores de acceso a Internet y empresas de contenido. Análisis de la cadena de valor. Economía Industrial, 340, 31-41. VARIAN, H.R. (2006).

Copying and Copyright: Los derechos de propiedad en la era de Internet, Economía industrial, 360, 17-27.

\section{Cita de este artículo}

LARRAÑAGA RUBIO, J. (2010) Economía de los contenidos online: periódicos online y ebooks. Revista Icono14 [en línea] 1 de Julio de 2010, Año 8, Vol. 2. pp. 0137. Recuperado (Fecha de acceso), de http://www.icono14.net

NOTA: Este trabajo ha recibido financiación del Proyecto Nacional del Ministerio de Ciencia e Innovación, referencia TEC 200607010/TCM 